\section{Monoklonale Antikörper und ihre Targets in der Therapie der Multiplen Sklerose}

\author{
A. Chan ${ }^{1}$ \\ 1 St. Josef-Hospital, Ruhr-Universität Bochum
}

Während unterschiedliche monoklonale Antikörper (mAk) in Indikationen wie der Rheumatologie oder Onkologie langjährig etabliert sind, sind in der Therapie der Multiplen Sklerose (MS) aktuell nur zwei mAk zugelassen (Natalizumab, Tysabri $^{\circledR}$; Alemtuzumab, Lemtrada ${ }^{\circledR}$; Stand September 2013). Gemessen an der Erwartung, hoch-spezifische, zielgerichtete Medikamente mit entsprechend geringen Nebenwirkungen zu erhalten, ist die bisherige Entwicklung von mAk für die verlaufsmodifizierende Therapie der MS mit teils gravierenden Rückschlägen und Einschränkungen behaftet gewesen. So führte der experimentell gut begründete, in der Rheumatologie erfolgreiche Ansatz einer TNF-alpha-Blockade (mit mAk oder Rezeptor-Fusionsprotein) überraschend zu einer Verschlechterung der Erkrankung bei MS-Patienten [1]. Mittlerweile sind demyelinisierende ZNS-Erkrankungen als Gegenanzeigen bzw. mögliche Nebenwirkungen einer anti-TNFalpha-Therapie gut bekannt. Dennoch bieten erst aktuellere Ergebnisse bezüglich MS-assoziierter genetischer TNF-Rezeptor-Varianten (TNFR1SF1A) mit Expression einer löslichen, TNF-alpha blockierenden TNFR1-Isoform eine plausible biologische Erklärung für die Verschlechterung einer Multiplen Sklerose unter anti-TNF-alpha-Therapie [2].

Auch initial erfolgreiche Entwicklungsprogramme sind von Rückschlägen nicht verschont geblieben. So war die Zeitspanne zwischen der Identifizierung des VLA-4 (very late antigen 4, $\alpha 4 / \beta 1$-Integrin Dimer) als relevantes leukozytäres Adhäsionsmolekül im Rahmen der Leukozytenmigration über die Blut-Hirnschranke bis zur Zulassung des humanisierten anti$\alpha 4$ mAk Natalizumab relativ kurz. Um so überraschender war kurz nach Zulassung des Natalizumab (Tysabri ${ }^{\circledR}$ ) das Bekanntwerden einer bis dahin in der Immuntherapie der MS unbekannten opportunistischen Infektion, der progressiven multifokalen Leukenzephalopathie (PML) [3]. Bei weltweit mittlerweile knapp 400 aufgetretenen PML-Fällen (Stand September 2013) und einer Letalität von ca. $20 \%$ beruhen bisherige Strategien zur Risikostratifizierung auf epidemiologischen Daten einerseits sowie dem spezifischen viralen Antikörper-Serostatus andererseits [4]. So ist das PML-Risiko für Patienten, die länger als 24 Monate mit Natalizumab behandelt werden, eine immunsuppressive Vortherapie erhalten haben sowie Antikörper gegen das verursachende JC-Polyoma-Virus aufweisen, bedeutsam erhöht (ca. 1:90) [5]. Diese Daten sind angesichts der bestehenden bzw. zur Zulassung anstehenden MS-Therapeutika bezüglich der Weiterentwicklung von Therapiealgorithmen (z.B. Reihenfolge, Zeitpunkt des Einsatzes) hoch relevant.

Während die Pathogenese der PML unter Natalizumab weiterhin ungeklärt ist, deuten PML-Fälle auch unter anderen therapeutischen mAk wie z.B. Efalizumab (anti CD11a, Pso- riasis) und Rituximab (anti CD20, rheumatoide Arthritis, systemischer Lupus erythematodes) [6] auf einen profunden Eingriff in immunbiologische Netzwerke trotz des jeweils vermuteten spezifischen Wirkmechanismus hin. Potentiell funktionell relevante, aber unzureichend verstandene Aspekte beinhalten hierbei die Funktion bzw. die zelluläre Expression des Zielantigens, die Antigen-Antikörper-Interaktion (bindend, inhibierend, signalauslösend), Antikörper Effektor Funktion (Zytotoxizität, Phagozytose) sowie die Gewebepenetration (u. a. über die Blut-Hirnschranke).

Ein weiteres Beispiel für eine hohe therapeutische Effektivität in Verbindung mit zuvor nicht antizipierten Nebenwirkungen stellt der humanisierte anti-CD52 IgG1-Antikörper Alemtuzumab dar. Initial für die Behandlung der chronisch lymphatischen Leukämie vom B-Zell Typ zugelassen, wurde dieses Medikament auf experimenteller Basis schon frühzeitig bei Autoimmunerkrankungen inkl. der MS angewandt. Das Glykoprotein CD52 wird dabei breit auf verschiedenen Immunzellen exprimiert, so dass das Alemtuzumab zu einer vermutlich großteils Komplement-vermittelten Depletion entsprechender Zielzellen (T-/B-Lymphozyten, NK-Zellen, dendritische Zellen, Monozyten/Makrophagen) führt. Aufgrund der unterschiedlichen Kinetik der Repopulation von Immunzellen ist das Repertoire nach der Alemtuzumab-Therapie deutlich verändert, was einerseits die hohe Effektivität bei hochaktiver MS erklären könnte, daneben aber auch eine Ursache der beobachteten Nebenwirkungen darstellen könnte. So sind teils schwerwiegende Autoantikörper-vermittelte Nebenwirkungen wie autoimmune Schilddrüsenerkrankungen, Immunthrombozytopenien (ITP), sowie autoimmune Basalmembranerkrankungen (Goodpasture Syndrom) im Rahmen des klinischen Entwicklungsprogrammes aufgetreten [7]. Während bezüglich der individuellen Nutzen-Risikoabwägung hohe Anforderungen an die Indikationsstellung, Durchführung und das Monitoring der Alemtuzumab-Therapie zu stellen sind, umfasst die Zulassung eine breitere Gruppe aktiver MS-Verläufe (Stand September 2013, Lemtrada ${ }^{\circledR}$ ). Eine solchermaßen frühzeitige, aggressive Immuntherapie hat deutliche Implikationen bezüglich gegenwärtiger Therapiealgorithmen.

Ein weiteres Zielmolekül stellt das CD20-Antigen auf der Oberfläche von Zellen der B-Zellreihe dar, welches aber nicht auf Antikörper-sezernierenden Plasmazellen exprimiert ist. Der chimärisierte anti-CD20 mAk Rituximab wurde ursprünglich für die Therapie des B-Zelllymphoms entwickelt, ist mittlerweile aber beispielsweise auch für die Behandlung der rheumatoiden Arthritis zugelassen. Nach Phase I- II-Studien in der MS sowie auch bei der Neuromyelitis optica sind individuelle Heilversuche („off lable use“) mit dieser Substanz auch in Deutschland verbreitet. Demhingegen fokussieren sich die klinisch fortgeschrittenen Entwicklungsprogramme aber auf humanisierte anti-CD20 Antikörper (Ocrelizumab, Ofatumumab).

Auch der sich gegenwärtig im fortgeschrittenen klinischen Entwicklungsprogramm befindliche Antikörper Daclizumab ist bereits aus anderen Indikationen (Transplantationsmedizin) bekannt. Dieser humanisierte IgG1-Antikörper blockiert CD25, welches das IL2-bindende Epitop der Alpha-Kette des IL2-Rezeptors darstellt. Während die initiale Rationale in der 
Entwicklung ein inhibierender Einfluss auf die Proliferation von Effektor-T-Zellen war, umfasst der Wirkmechanismus aber pleiotrope Effekte, wie eine Expansion potentiell regulatorischer Zellen (CD56 bright natürliche Killerzellen). Daneben hat die Untersuchung weiterer potentieller Wirkmechanismen des Daclizumab vertiefte Einblicke in die frühe T-Zellaktivierung durch dendritische Zellen erbracht. Somit stellt auch das Daclizumab ein gutes Beispiel dafür dar, dass der letztlich immunologisch dominierende Effekt einer zunächst spezifischen Antikörper-Blockade nicht gut vorhersagbar ist. Zusammengefasst zeigen mAk mit hochspezifischen und teils deutlich divergierenden Zielstrukturen erstaunlich robuste klinische Effekte in der Therapie der Multiplen Sklerose. Dies mag zumindest teilweise in den profunden Eingriffen in breitere immunbiologische Netzwerke durch die unterschiedlichen Substanzen begründet sein. Dies beinhaltet aber auch die Möglichkeit auch aus dem Tiermodell nicht vorhersagbarer Nebenwirkungen. Die Komplexität pleiotroper Interaktionen wird vermutlich in den nächsten Jahren noch steigen, da über derzeitige mAk mit immunologischen Zielstrukturen weitere, potentiell in der Remyelinisierung beteiligte Mechanismen in den Blickpunkt des Interesses rücken (z.B. Blockade von LINGO-1, leucine-rich repeat and Ig domain containing NOGO receptor interacting protein-1) [8].
Interessenkonflikt: Der Autor erhielt und erhält Forschungsunterstützungen von folgenden Firmen: Bayer Vital, Biogen Idec, Merck Serono, Novartis Pharma.

Der Autor ist als Berater oder Referent für folgende Firmen tätig oder tätig gewesen: Allergan, Allmirall, Bayer Vital, Biogen Idec, Genzyme, Merck Serono, Novartis Pharma, Sanofi-Aventis, Teva.

\section{Literatur}

1 Bosch X et al. Nature Rev Neurol 2011; 7: 165-171

2 Gregory AP et al. Nature 2012; 488: 508

3 Wenning W et al. New Engl J Med 2009; 361: 1075-1080

4 Trampe A et al. Neurology 2012; 78: 1736-1742

5 Bloomgren G et al. New Engl J Med 2012; 366: 1870-1880

6 Haghikia A et al. PLOSone 2011; 6: e18506

7 Coles AJ. Neurotherapeutics 2013; 10: 29-33

8 Mi S et al. CNS Drugs 2013; 27: 493-503

\section{Bibliografie}

Dol http://dx.doi.org/10.1055/s-0033-1346710

Drug Res 2013; 63, Suppl. 1: S10-S11

(c) Georg Thieme Verlag KG Stuttgart · New York .

ISSN 2194-9379 